\title{
Domain Walls and Matter-Antimatter Domains in the Early Uni- verse
}

\author{
A.D. Dolgov ${ }^{1,2, \star}$, S.I. Godunov ${ }^{1,2, \star \star}$, and A.S. Rudenko ${ }^{1,3, \star \star \star}$ \\ ${ }^{1}$ Novosibirsk State University, Novosibirsk, 630090, Russia \\ ${ }^{2}$ Institute for Theoretical and Experimental Physics, Moscow, 117218, Russia \\ ${ }^{3}$ Budker Institute of Nuclear Physics, Novosibirsk, 630090, Russia
}

\begin{abstract}
We suggest a scenario of spontaneous (or dynamical) $C$ and $C P$ violation according to which it is possible to generate domains of matter and antimatter separated by cosmologically large distances. Such $C(C P)$ violation existed only in the early universe and later it disappeared with the only trace of generated matter and antimatter domains. So this scenario does not suffer from the problem of domain walls.

According to this scenario the width of the domain wall should grow exponentially to prevent annihilation at the domain boundaries. Though there is a classical result obtained by Basu and Vilenkin that the width of the wall tends to the one of the stationary solution (constant physical width). That is why we considered thick domain walls in a de Sitter universe following paper by Basu and Vilenkin. However, we were interested not only in stationary solutions found therein, but also investigated the general case of domain wall evolution with time. When the wall thickness parameter, $\delta_{0}$, is smaller than $H^{-1} / \sqrt{2}$, where $H$ is the Hubble parameter in de Sitter space-time, then the stationary solutions exist, and initial field configurations tend with time to the stationary ones. However, there are no stationary solutions for $\delta_{0}>H^{-1} / \sqrt{2}$. We have calculated numerically the rate of the wall expansion in this case and have found that the width of the wall grows exponentially fast for $\delta_{0} \gg H^{-1}$. An explanation for the critical value $\delta_{0 c}=H^{-1} / \sqrt{2}$ is also proposed.
\end{abstract}

\section{Introduction}

Our local cosmological neighborhood is made of baryons, while fraction of antimatter is vanishingly small. Therefore, it looks like the universe is $100 \%$ baryo-asymmetric (at least locally). The Standard Model (SM) cannot explain this asymmetry (BAU). Many quite different extensions of the SM and various scenarios for the BAU generation were suggested in the literature, for a review see e.g. Refs. [1-5]. Typically, consideration is restricted to the models where the universe is asymmetric globally. However, it is not excluded that the real universe may be globally symmetric. It may consist of domains of matter and antimatter, and if the domains are sufficiently large and far away, they may escape observational constraints on matter-antimatter annihilation at the domain boundaries. In the

\footnotetext{
^e-mail: dolgov@fe.infn.it

$\star \star$ e-mail: sgodunov@itep.ru

$\star \star \star$ e-mail: a.s.rudenko@inp.nsk.su
} 
simplest version of the scenario the distance to the nearest domain of antimatter should be close to the present day cosmological horizon [6].

Corresponding particle physics models, leading to the universe creation with abundant antimatter domains were suggested and developed in the past. While being more involved, the models of this type also suffer from the inherent problem - a domain wall problem [7]. However, there is a way out of this restriction: domain walls should exist only in the past and should disappear by now, though their effects in the form of matter and antimatter objects would survive to the present day.

One class of models where this can be achieved has been suggested in Refs. [8-11]. The main idea behind is a possibility of an unusual symmetry behavior at high temperatures. It is well known that a symmetry, which is broken in vacuum, at high temperatures tends to be restored. But in general, it is also possible for a symmetry to be broken only in a specific range of temperatures, for the particular models and details see [8-11]. This is just what is needed for a matter-antimatter domain generation without domain wall problem. However, if a model is based on the unusual symmetry behavior at high temperatures, then the size of domains will be too small from the cosmological point of view. We suggest the scenario in which this happens during inflationary stage. Domains with different sign of $C P$ disappear by now, so the domain wall problem is absent. However, they appeared during inflation and survived at the baryogenesis epoch, therefore, cosmologically large ${ }^{1}$ domains of matter and antimatter could be created.

The scenario described above inspired the consideration of the related problem: is it possible for the domain wall width to grow almost at exponential rate in expanding universe? The evolution of the domain walls in de Sitter space-time was considered by Basu and Vilenkin [12]. The authors argued that the width of the domain wall is determined by the ratio $C \equiv \lambda \eta^{2} / H^{2}>0$, where $H$ is the Hubble parameter, which was assumed to be constant, $\eta$ is the vacuum expectation value of the Higgs-like field which induced the spontaneous symmetry breaking, and $\lambda$ is the coupling constant in the doublewell potential, see (17). If $C \gg 2$, the width of the domain wall would be close to its flat space-time value, $\delta_{0}=1 /(\sqrt{\lambda} \eta)$, which is microscopically small.

On the other hand, if $C<2$, it is not excluded that the width of the domain wall may be astronomically large. We show that this is indeed the case. In ref. [12] only the stationary problem was considered, when the shape of the domain wall was a function of a single variable, the physical distance. In the paper [12] it was found numerically that the stationary solution exists only if $C>2$.

In what follows we lift the assumption of the stationarity and consider the general plane solution being a function of both variables: the distance from the wall and time. It allows us to see how the solution approaches the stationary one and, in particular, what happens with initial configurations if $C \leq 2$, when the stationary solution does not exist. The related problems were considered in [13].

This talk is based on papers [14] (written in collaboration with I.I. Tkachev) and [15].

\section{Generation of matter and animatter domains}

\subsection{Model}

In the suggested model the difference between matter and antimatter is generated by pseudoscalar field $\chi$ which interacts with inflaton field $\Phi$. We assume the following Lagrangian:

$$
L=L_{\Phi}+L_{\chi}+L_{i n t}
$$

\footnotetext{
${ }^{1}$ The matter-antimatter domains should be separated by at least several megaparsec in terms of the present day scale to avoid excessive matter-antimatter annihilation. On the other hand, the distance should not be too large, otherwise the scenario would lead to too large angular fluctuations of CMB [6].
} 

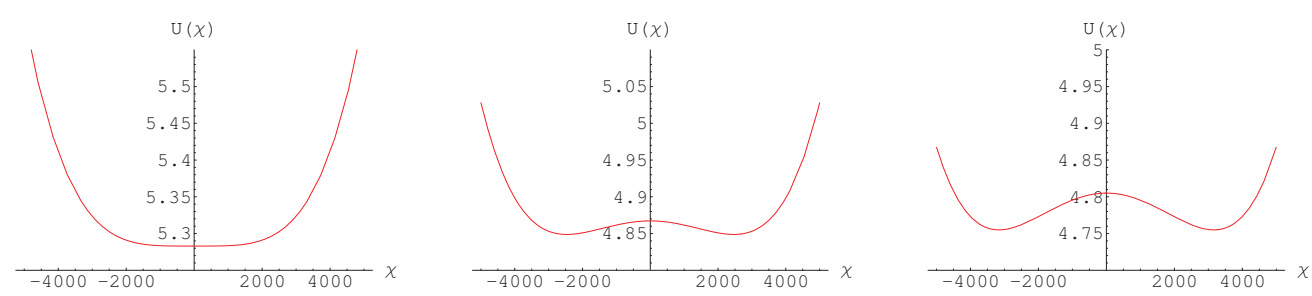

Figure 1. Potential $U(\Phi, \chi)$ at constant $\Phi$. The left plot corresponds to the moment when the effective mass of $\chi$ becomes zero, i.e. when $m^{2} / 2-\mu^{2} V(\Phi)=0$, or $\Phi=\Phi_{0}+2 \Phi_{1} \sqrt{\ln (\sqrt{2} \mu / m)}$. The middle and the right plots show the moments when $\Phi=\Phi_{0}+\Phi_{1}$ and $\Phi=\Phi_{0}$, respectively. The parameters are $\Phi_{0}=3.1 m_{P l}, \Phi_{1}=0.02 m_{P l}$, $\mu=10^{-4} m_{P l}$ and $m=10^{-10} m_{P l}$. Field $\chi$ is measured in units of $M$ and $U(\Phi, \chi)$ is measured in units of $10^{-12} \mathrm{~m}_{P l}^{4}$.

where

$$
L_{\Phi}=\frac{1}{2}(\partial \Phi)^{2}-\frac{1}{2} M^{2} \Phi^{2}, \quad L_{\chi}=\frac{1}{2}(\partial \chi)^{2}-\frac{1}{2} m^{2} \chi^{2}-\frac{1}{4} \lambda_{\chi} \chi^{4}, \quad L_{i n t}=\mu^{2} \chi^{2} V(\Phi) .
$$

Here the metric tensor enters into kinetic terms in the usual way, and $M, m, \lambda_{\chi}, \mu$ are some constant parameters. The dimensionless function $V(\Phi)$ is chosen in the way that it is non-zero only when $\Phi$ is close to some constant value $\Phi_{0}$. In this paper we choose it as Gaussian function

$$
V(\Phi)=\exp \left[-\frac{\left(\Phi-\Phi_{0}\right)^{2}}{2 \Phi_{1}^{2}}\right]
$$

Parameters $\Phi_{0}$ and $\Phi_{1}$ have dimension of mass and indicate position of the "bell" center and characteristic width of the "bell", respectively.

The equations of motion have the form:

$$
\begin{gathered}
\ddot{\Phi}+3 H \dot{\Phi}+M^{2} \Phi+\mu^{2} \chi^{2} \frac{\Phi-\Phi_{0}}{\Phi_{1}^{2}} V(\Phi)=0, \\
\ddot{\chi}+3 H \dot{\chi}+m^{2} \chi+\lambda_{\chi} \chi^{3}-2 \mu^{2} \chi V(\Phi)=0,
\end{gathered}
$$

where $H=\dot{a} / a$ is the Hubble parameter, $a(t)$ is the cosmological scale factor which enters into the FLRW metric as

$$
d s^{2}=d t^{2}-a^{2}(t) d \mathbf{x}^{2}
$$

It is assumed here that fields $\Phi$ and $\chi$ depend only on time, $\Phi=\Phi(t)$ and $\chi=\chi(t)$.

The Hubble parameter is expressed through the energy density $\rho$ as

$$
H=\sqrt{\frac{8 \pi \rho}{3 m_{P l}^{2}}}=\sqrt{\frac{8 \pi}{3 m_{P l}^{2}}\left(\frac{\dot{\Phi}^{2}}{2}+\frac{M^{2} \Phi^{2}}{2}+\frac{\dot{\chi}^{2}}{2}+\frac{m^{2} \chi^{2}}{2}+\frac{\lambda_{\chi} \chi^{4}}{4}-\mu^{2} \chi^{2} V(\Phi)\right)},
$$

where $m_{P l} \approx 1.2 \cdot 10^{19} \mathrm{GeV}$ is the Planck mass.

The interaction introduced above leads to the following scenario. During inflation the magnitude of the inflaton field $\Phi$ decreases and when it reaches vicinity of $\Phi_{0}$ two minima appear in the potential

$$
U(\Phi, \chi)=\left(\frac{1}{2} m^{2}-\mu^{2} V(\Phi)\right) \chi^{2}+\frac{1}{4} \lambda_{\chi} \chi^{4}+\frac{1}{2} M^{2} \Phi^{2}
$$


at constant $\Phi$, so the point $\chi=0$ becomes local maximum (see Fig. 1).

Therefore, in the spatial regions where the field $\chi$ turns out to be positive (due to fluctuations) it rolls down to the positive minimum $\eta>0$, and in the regions where $\chi$ is negative it goes to the negative minimum $-\eta<0$. Thus one can substitute $\chi=\tilde{\chi}+\eta$ and $\chi=\tilde{\chi}-\eta$, respectively, where $\eta$ and $-\eta$ have the meaning of the vacuum expectation values $\langle\chi\rangle$. Thus, due to initial fluctuations we should obtain domains of the field $\chi$ which will provide the source of $C P$ violation, so in the domains with positive $\langle\chi\rangle$ we will have domain of matter while in the domain with negative $\langle\chi\rangle$ we will have domain of antimatter. After the interaction term $V(\Phi)$ is switched off there is again single minimum $\chi=0$ so there is no domain walls problem at the present time.

We expect that the distances between domains, as well as domain sizes exponentially grow with the scale factor $a(t)$, so at the present time these domains are of cosmological size and they are separated by large distance which prevents the annihilation at their boundaries. According to [6], a very large piece of space between the domains, devoid of baryons, would lead to too large angular fluctuations of the CMB temperature. On the other hand, if the distance between domains is smaller than the baryon diffusion length, they would be able to meet successfully their counterparts and to annihilate. These arguments resulted in the conclusion that the nearest domains should be at the cosmologically large distance from us, about a few Gpc. On the other hand, the effects of the cosmological inhomogeneity, not yet studied, could inhibit the baryon diffusion and would relax the bound on the distance to the nearest antimatter domain.

Now let us consider what happens at different stages of the scenario, so we will be able to put the limits on initial conditions and parameters of the model.

For simplicity we assume, though it is not necessary, that the impact of field $\chi$ on cosmological expansion at inflationary stage is negligible. To this end we suppose that the energy density of $\Phi$ dominates over that of $\chi$ and the energy density of their interaction. In this approximation the second derivative term and the interaction term proportional to $V(\Phi)$ in the inflaton equation of motion (4) can be neglected. Therefore, we find

$$
\dot{\Phi}=-\frac{M^{2} \Phi}{3 H}, \quad \Phi(t)=\Phi_{i n}-\frac{M m_{P l} t}{2 \sqrt{3 \pi}},
$$

where $\Phi_{\text {in }}$ is an initial value of $\Phi$. In this scenario of inflation the Hubble parameter gradually decreases, $H \sim \Phi$, and to obtain a sufficiently long inflation it is sufficient to take $\Phi_{\text {in }} \gtrsim 3.3 \mathrm{~m}_{P l}$.

In order to avoid too large density perturbations one should choose the value of the inflaton mass $M$ in the range $10^{-7} \lesssim M / m_{P l} \lesssim 10^{-6}$; accordingly we take $M=10^{-6} m_{P l}$.

To arrange the desired scenario we should set the value of $\Phi_{0}$ still at inflationary stage such that the distances between different domains and the domain sizes exponentially expanded up to cosmologically large scales. To ensure it we should take $\Phi_{0} \gtrsim 3.1 \cdot m_{P l}$ (see [14] for details).

Depending upon the value of $\Phi$, there are three different regimes of the evolution of the field $\chi$ :

1. Initial stage: $\Phi>\Phi_{0}$ and $\left(\Phi-\Phi_{0}\right) \gg \Phi_{1}$.

The mass of $\chi$ is supposed to be small in comparison with the expansion rate, $m<H$, so field $\chi$ sits near the minimum of its potential at $\chi=0$ (see left plot in Fig. 1).

2. Second stage: $\left|\Phi-\Phi_{0}\right| \lesssim \Phi_{1}$.

At this stage the squared effective mass of $\chi$ becomes negative. The minima of the potential (8) move from zero as $\eta(t)$ or $-\eta(t)$, where $\eta^{2}(t)=\frac{1}{\lambda_{\chi}}\left(2 \mu^{2} V(\Phi)-m^{2}\right)$. To have equal amplitudes of both positive and negative $C P$ violation we need to impose the condition that $\chi$ reaches one or other minimum $\chi= \pm \eta(t)$ and stays there (approximately). This minimum moves 
exponentially fast and $\chi$ would follow it e.g. if $\mu \gg H$ (since in this case $\chi \propto \exp (\mu t)$ ). For $M=10^{-6} m_{P l}, \Phi_{0}=3.1 m_{P l}$ we get $\mu \gg H=\sqrt{4 \pi / 3} M / m_{P l} \Phi \sim 6 \cdot 10^{-6} m_{P l}$.

But even when $\chi$ grows exponentially there still should be enough time for $\chi$ to reach the minimum $\eta(t)$. If the inflaton $\Phi$ goes from $\Phi_{0}+2 \Phi_{1}$ to $\Phi_{0}-2 \Phi_{1}$ during the interval $\tau$, we should require that

$$
\mu \tau=\mu \frac{8 \sqrt{3 \pi} \Phi_{1}}{M m_{P l}} \gtrsim \ln \frac{\eta_{\max }}{\chi_{\text {in }}}=\frac{1}{2} \ln \frac{2 \mu^{2}}{\lambda_{\chi} \chi_{\text {in }}^{2}} \Rightarrow \mu \gtrsim \frac{M m_{P l}}{16 \sqrt{3 \pi} \Phi_{1}} \ln \frac{2 \mu^{2}}{\lambda_{\chi} \chi_{\text {in }}^{2}},
$$

where $\eta_{\max } \equiv 2 \mu^{2} / \lambda_{\chi}$ and $\chi_{\text {in }}$ is initial value of $\chi$.

According to this scenario $\chi^{2} \sim \eta_{\max }^{2} \equiv 2 \mu^{2} / \lambda_{\chi}$ is the largest value of $\chi^{2}$. So to be sure that the inflaton field $\Phi$ always gives the main contribution to the energy density, we should impose the conditions:

$$
\left.M^{2} \Phi_{0}^{2} \gg \mu^{2} \chi^{2}\right|_{\Phi=\Phi_{0}} \sim \frac{\mu^{4}}{\lambda_{\chi}} \Rightarrow \mu^{4} \ll M^{2} \Phi_{0}^{2} \lambda_{\chi}
$$

For $M=10^{-6} m_{P l}, \Phi_{0}=3.1 m_{P l}$ we get $\mu \ll 1.8 \cdot 10^{-3} m_{P l} \sqrt[4]{\lambda_{\chi}}$.

After $\Phi$ passed $\Phi_{0}, \eta(t)$ started to decrease exponentially and turned zero when $\Phi=\Phi_{0}-$ $2 \Phi_{1} \sqrt{\ln (\sqrt{2} \mu / m)}$, so the potential (8) started to have only one minimum at $\chi=0$ again. The field $\chi$ also began to decrease rapidly until $V(\Phi)$ became small in comparison with other terms in Eq. (5).

3. Final stage: $\Phi<\Phi_{0}$ and $\left(\Phi_{0}-\Phi\right) \gg \Phi_{1}$.

When the interaction term $\mu^{2} \chi^{2} V(\Phi)$ vanishes, the evolution of field $\chi$ would be determined by the terms with $\lambda_{\chi}$ and $m$, see Eq. (5). If $\lambda_{\chi}$ is not small enough and $\chi$ is still large then the term $\lambda_{\chi} \chi^{3}$ dominates and the equation of motion turns into $3 H \dot{\chi}+\lambda_{\chi} \chi^{3}=0$, which has the solution $\chi=\sqrt{\frac{3 H}{2 \lambda_{\chi}}} \frac{1}{\sqrt{t-C}}$, where $C$ is a constant of integration. We see that in this regime $\chi$ decreases as a power of $t$.

When $\chi$ becomes quite small, Eq. (5) turns into $\ddot{\chi}+3 H \dot{\chi}+m^{2} \chi=0$, so the field $\chi$ slowly oscillates and decreases due to redshift related to cosmological expansion.

\subsection{Numerical calculations}

In order to check that the described scenario is indeed operative we performed the numerical calculations in the homogeneous case with the following set of parameters (all dimensional values are given in the Planck mass, $m_{P l}$, units):

$$
\Phi_{i n}=4, \Phi_{0}=3.1, \Phi_{1}=0.02, M=10^{-6}, \chi_{i n}=10^{-6}, m=10^{-10}, \lambda_{\chi}=2 \cdot 10^{-3}, \mu=10^{-4} \text {. }
$$

In Figs. 2 and 3 the results of numerical simulation are shown. One can see that the interaction of the inflaton field $\Phi$ with the field $\chi$ does not break the standard inflation, $\Phi(t)$ only slightly deviates from straight line around $\Phi=\Phi_{0}=3.1 \mathrm{~m}_{P l}$.

In Fig. 3 one can see how the field $\chi$ rolls down to the minimum of the potential $\eta(t)>0$ and oscillates there. Then it goes back to the old minimum at $\chi=0$, but even at the end of inflation $\chi$ remains an order of magnitude larger than it was at the beginning $\left(\chi \sim 10 M\right.$ at $t \approx 25 M^{-1}$, while $\chi_{\text {in }}=1 M$ ). At the moment $t \approx 35 M^{-1}$ the field $\chi$ crosses zero and starts slow oscillations with very low frequency, which could be much lower than the characteristic rate of baryogenesis. 

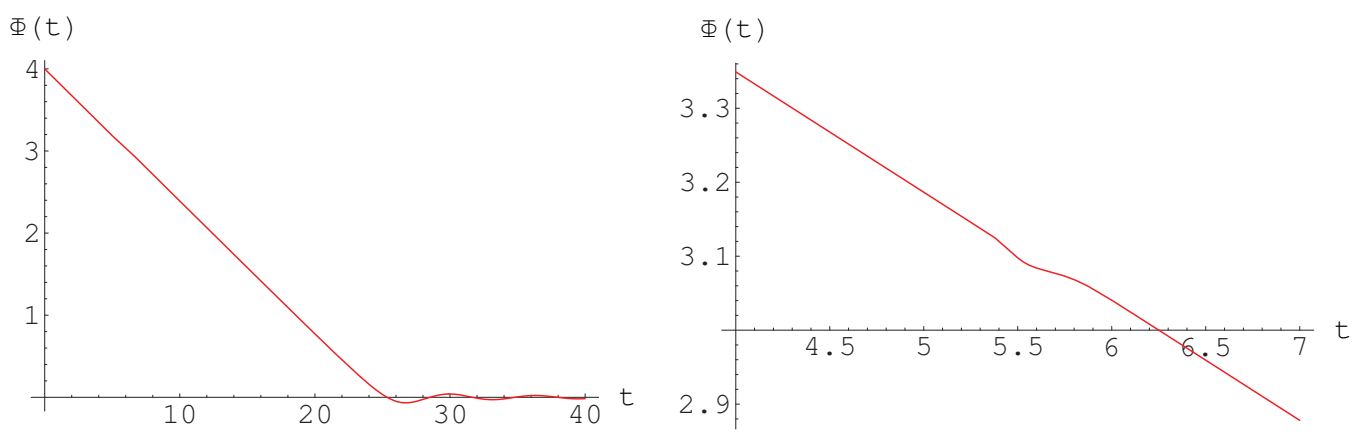

Figure 2. Time evolution of the inflaton field $\Phi(t)$ for the parameters (12). Time $t$ is measured in units of $M^{-1}$ and $\Phi$ is measured in units of $m_{P l}$. The right plot shows the evolution of $\Phi$ in more detail near the point $\Phi=\Phi_{0}=3.1 \mathrm{~m}_{P l}$.
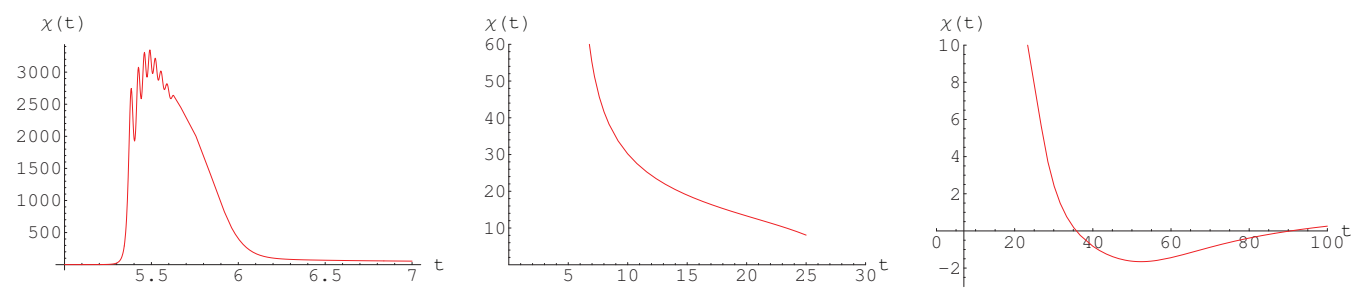

Figure 3. Time dependence of the field $\chi(t)$ for the parameters (12). Time $t$ is measured in units of $M^{-1}$ and $\chi$ is measured in units of $M$. All the three plots depict the evolution of $\chi$ during different time intervals. In the middle and right plots one can see that at the end of inflation, $t \approx 25 M^{-1}$, the field $\chi$ remains quite large.

So we see that parameters (12) are chosen in a proper way. In this model the influence of $\chi$ on the process of inflation is insignificant, thus the domain size grows exponentially. The remained question is how the distance between domains grows. The related problem of domain wall width in expanding universe is considered in the Section 3.

\subsection{Production of heavy particles after inflation and baryogenesis}

As it is commonly known, the stage of inflation is followed by the stage of (re)heating, during which the very heavy $X$ particles can be produced through decay of inflaton field (see e.g. book [16]) or from vacuum fluctuations in gravitational field [17].

Let us consider the situation when the inflaton field $\Phi$ interacts with $X$ particles (for example, scalar bosons) through the coupling $g_{X}^{2} \Phi^{2} X^{2}$. As is shown in Refs. [18-20], the particle production can be strongly enhanced due to parametric resonance. For an unsuppressed production it is essential that the resonance is broad, which is true for the wide range of coupling constant, $g_{X} \gg 4 \cdot 10^{-6}$. Because of resonance, very heavy $X$-bosons with masses even of GUT scale $m_{X} \sim\left(M m_{P l}\right)^{1 / 2} \sim 10^{16}$ $\mathrm{GeV}$ can be produced under quite reasonable assumption $g_{X} \sim 1$ [16]. Moreover in this case of comparatively large coupling constant $g_{X} \sim 1$ the decay of the inflaton field occurs very rapidly, during only one or few oscillations. 
Let us assume that the produced $X$-bosons in turn decay into fermions, for example, into quarkquark and antiquark-antilepton pairs, $X \rightarrow q q$ and $X \rightarrow \bar{q} \bar{l}$, respectively. If the corresponding coupling constants are large enough, the $X$-bosons decay very quickly. Therefore, the field $\chi(t)$ may remain non-zero yet to the moment when $X$-bosons have been completely decayed, indeed $\chi \sim 2 M \sim 10^{13}$ $\mathrm{GeV}$ at $t=30 \mathrm{M}^{-1}$ (see Fig. 3).

We want to provide the source of $C P$ violation for this mechanism via the introduced field $\chi$. It can be done if we suppose that $\chi$ interacts with the produced fermions as

$$
L_{\chi \psi \psi}=g_{k l} \chi \bar{\psi}^{k} i \gamma_{5} \psi^{l}=g_{k l} \chi\left(\bar{\psi}_{R}^{k} i \gamma_{5} \psi_{L}^{l}+\bar{\psi}_{L}^{k} i \gamma_{5} \psi_{R}^{l}\right)=i g_{k l} \chi\left(\bar{\psi}_{L}^{k} \psi_{R}^{l}-\bar{\psi}_{R}^{k} \psi_{L}^{l}\right),
$$

where $k$ and $l$ denote the fermion flavor (sum over repetitive indices is assumed), $\psi_{R}=\left(\left(1+\gamma_{5}\right) / 2\right) \psi$, $\psi_{L}=\left(\left(1-\gamma_{5}\right) / 2\right) \psi$. Since $\chi$ is supposed to be electrically neutral, it interacts with quarks with the same electric charge, so $k$ and $l$ either run over $u, c, t$ or $d, s, b$ and there are no cross terms.

The interaction of fermions with pseudoscalar field $\chi$ can be "rotated away" two unitary transformations $\psi_{R} \rightarrow \psi_{R}^{\prime}=U_{R} \psi_{R}$ and $\psi_{L} \rightarrow \psi_{L}^{\prime}=U_{L} \psi_{L}$. However, the interaction of fermions with vector (gauge) boson $X$ remains the same under these transformations:

$$
g_{R k l} X_{\mu} \bar{\psi}_{R}^{k} \gamma^{\mu} \psi_{R}^{l}+g_{L k l} X_{\mu} \bar{\psi}_{L}^{k} \gamma^{\mu} \psi_{L}^{l} \rightarrow g_{R a b}^{\prime} X_{\mu} \bar{\psi}_{R}^{a} \gamma^{\mu} \psi_{R}^{b}+g_{L a b}^{\prime} X_{\mu} \bar{\psi}_{L}^{a} \gamma^{\mu} \psi_{L}^{b},
$$

here $g_{R}^{\prime}=U_{R} g_{R} U_{R}^{\dagger}$ and $g_{L}^{\prime}=U_{L} g_{L} U_{L}^{\dagger}$ are matrices of coupling constants in mass eigenstate basis, so $\psi^{a}$ describes $a$-th sort of fermion with definite mass. The constants $g_{a b}^{\prime}$ are complex in general case, and if there are at least three species of fermions, one cannot rotate away simultaneously all phases in complex matrices $g_{R, L}^{\prime}[21]$. The complexity of the coupling constants means that $C P$ is violated in the $X$-boson decays [22]. The magnitude of this $C P$ violation depends on the value of the field $\chi$ through the matrices $U_{R, L}$ and coupling constants $g_{R, L}^{\prime}$. Since $\chi$ is essentially non-zero after the end of inflation and during baryogenesis, the $C P$-odd effects can be large enough.

We assume also that gauge interactions involve fermions with certain chirality, see (14), and thus these interactions break $C$-invariance.

$C$ and $C P$ violation is one of the necessary Sakharov conditions of generation of baryon asymmetry [23]. The another one is baryon number violation, so one needs to assume also that in the decays of $X$-bosons the baryon number is not conserved. Let $\delta$ be the baryon asymmetry generated in the decay of one $X$-boson. Then it can be easily demonstrated [16] that the ratio of the baryon number density to the entropy density is estimated as

$$
\Delta_{B}=\frac{n_{B}-n_{\bar{B}}}{s} \sim \delta \frac{h}{g_{X}}\left(\frac{m_{t h}}{m_{P l}}\right)^{1 / 2},
$$

where $h$ and $g_{X}$ are typical coupling constants of $X$-boson with fermions and inflaton, respectively, $m_{t h}$ is mass scale of the theory. It is quite reasonable to believe that $h / g_{X} \sim 1$ and $m_{t h} \sim M \sim 10^{-6} m_{P l}$, so one has $\Delta_{B} \sim 10^{-3} \delta$. Thus, to get observed value $\Delta_{B} \simeq 0.86 \cdot 10^{-10}$ it is sufficient to have only $\delta \sim 10^{-7}$. Such small $\delta$ seems to be easily produced in the decay of $X$-boson. Therefore, the observed baryon asymmetry of the universe can be generated in the decays of $X$-bosons, without fine tuning of parameters of the theory.

\section{Domain walls in expanding universe}

\subsection{Stationary solutions}

In spatially flat section of de Sitter universe the expansion rate, $H=\dot{a} / a$, is constant and the scale factor evolves as $a(t)=\exp H t$. The FLRW metric for such universe has the form:

$$
d s^{2}=d t^{2}-e^{2 H t}\left(d x^{2}+d y^{2}+d z^{2}\right)
$$


Let us consider a model of real scalar field $\varphi$ with the Lagrangian

$$
\mathcal{L}=\frac{1}{2} g^{\mu \nu} \partial_{\mu} \varphi \partial_{\nu} \varphi-\frac{\lambda}{2}\left(\varphi^{2}-\eta^{2}\right)^{2}
$$

The corresponding equation of motion is

$$
\frac{1}{\sqrt{-g}} \partial_{\mu}\left(\sqrt{-g} g^{\mu v} \partial_{\nu} \varphi\right)=-2 \lambda \varphi\left(\varphi^{2}-\eta^{2}\right) .
$$

In flat space-time, $H=0$, and in one-dimensional static case, $\varphi=\varphi(z)$, the equation takes the form $\frac{d^{2} \varphi}{d z^{2}}=2 \lambda \varphi\left(\varphi^{2}-\eta^{2}\right)$ and has a kink-type solution, which describes a static infinite domain wall. Without loss of generality we can assume that the wall is situated at $z=0$ in $x y$-plane:

$$
\varphi(z)=\eta \tanh \frac{z}{\delta_{0}},
$$

where $\delta_{0}=1 /(\sqrt{\lambda} \eta)$ has the meaning of the wall thickness (subscript 0 indicates that $H=0$ ).

Now let us consider an expanding universe with constant $H>0$. In this case, if one looks for stationary solution (see [12]), it is reasonable to suggest that the field $\varphi$ depends only on $z a(t)=$ $z \exp H t$, which is the proper distance from the wall. So, one can choose the following ansatz for $\varphi$ :

$$
\varphi=\eta \cdot f(u), \quad \text { where } u=H z e^{H t}
$$

where $u$ and $f$ are dimensionless. Then the equation of motion takes the form:

$$
\left(1-u^{2}\right) f^{\prime \prime}-4 u f^{\prime}=-2 C f\left(1-f^{2}\right) \text {. }
$$

Here prime means the derivative with respect to $u$. It is noteworthy that all parameters of the problem are combined into a single positive constant $C=1 /\left(H \delta_{0}\right)^{2}=\lambda \eta^{2} / H^{2}>0$.

Since we are interested in kink-type solutions, the boundary conditions should be

$$
f(0)=0, \quad f( \pm \infty)= \pm 1 .
$$

Corresponding numerical solutions for different values of parameter $C$ are shown in Fig. 1 of $[12,15]$. We see that the larger is $C$ the closer is the solution to the flat space-time one $(H=0)$, as is naturally expected.

As it is noticed in ref. [12] the stationary solutions can be found only for $C>2$, but no explanation of this observation is given therein. For the detailed explanation see [15].

\subsection{Evolution of domain walls beyond the stationary limit}

As we have seen in the previous section, Eq. (21) allows to find the field configurations, which describe stationary domain walls in expanding universe. However, it is also interesting to see how domain walls evolve from some initial states. Beyond the stationary approximation we can find not only solution for $C>2$ but also for $C \leq 2$, for which the stationary approximation does not exist.

To this end one should solve the original equation of motion (18) in the case when the field $\varphi$ is a function of two independent variables, $z$ and $t$. It is convenient to introduce dimensionless variables $\tau=H t, \zeta=H z$ and function $f(\zeta, \tau)=\varphi(z, t) / \eta$. As a result one obtains the equation

$$
\frac{\partial^{2} f}{\partial \tau^{2}}+3 \frac{\partial f}{\partial \tau}-e^{-2 \tau} \frac{\partial^{2} f}{\partial \zeta^{2}}=2 C f\left(1-f^{2}\right)
$$



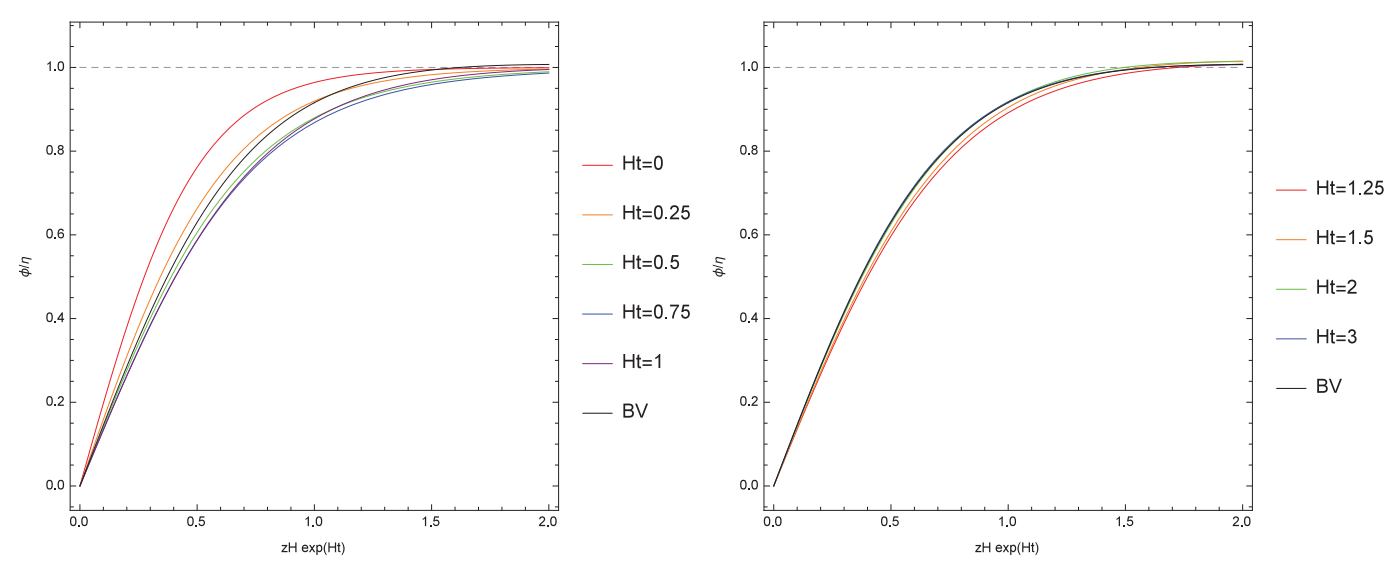

Figure 4. Evolution of domain wall for $C=4$. Black curve corresponds to stationary solution.

where $C=\lambda \eta^{2} / H^{2}=1 /\left(H \delta_{0}\right)^{2}>0$ as it was above.

The boundary conditions for the kink-type solution should be

$$
f(0, \tau)=0, \quad f( \pm \infty, \tau)= \pm 1
$$

and we choose the initial configuration as the domain wall with "natural" thickness $1 / \sqrt{C}$ (with respect to dimensionless coordinate $\zeta$ ) and zero time derivative. Of course, this is a toy model with the artificial initial conditions. The realistic model should describe the evolution of domain walls from the very beginning, including the process of wall formation. This will be studied elsewhere.

The evolution of the domain wall for $C=4$ is depicted in Fig. 4. The stationary solution is shown by black curve (it is denoted by "BV" because of Basu and Vilenkin who found it [12]). One sees that the domain wall evolves in somewhat non-trivial way. At the very beginning the wall starts to broaden in terms of the proper distance from the wall, $z H \operatorname{exp~} H t$, and at some moment it becomes wider than the stationary solution. However, afterwards the wall broadening changes to contraction. Finally, the wall comes to the stationary configuration after several damped oscillations around it.

When $C$ is close to 2, the stationary solution is very broad and the solution of Eq. (23) converges to the stationary one very slowly. Unlike using plots like in Fig. 4, it is more convenient to look at the evolution of the wall width with time. At the initial moment in our model the wall is described by hyperbolic tangent. Although in the realistic course of the evolution the wall is not "pure" hyperbolic tangent, nevertheless one can use the same definition for the thickness $\delta(t)$ as the value of the coordinate $z$ at which the field $\phi$ reaches the value $\phi / \eta=\tanh 1 \approx 0.76$.

In Fig. 5 the time dependence of the physical width of the wall for different values of $C$ is presented. In the left plot $(C>2)$ one can see that all the curves indeed tend to constant values corresponding to stationary solutions (dashed lines). Oscillatory behavior mentioned above is also apparent. Right plot contains curves for $C<2$. It is clear that for $C=1$, i.e. for not very small values of $C$, the wall thickness increases slower than exponent. However, for smaller values of $C$, e.g. $C \lesssim 0.1$ the rate of the wall expansion is the exponential one with a good accuracy.

The Hubble constant, $H$, plays the role of friction in this problem and when it dominates over the potential term $(C \ll 1)$, the field configuration as a function of coordinates becomes almost static. Therefore the width of the wall is growing nearly as the scale factor $a(t)$, i.e. almost exponentially. This is why there are no stable solutions for $C \ll 1$. 

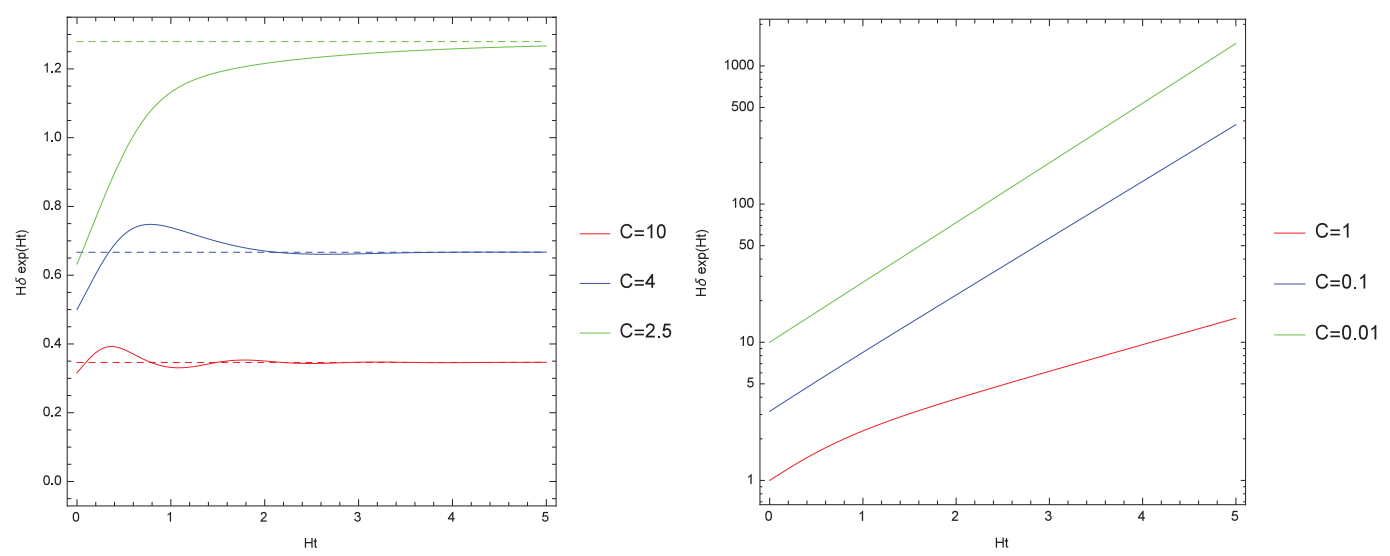

Figure 5. Time dependence of domain wall thickness for $C>2$ (left plot) and $C<2$ (right plot). Dashed lines describe the stationary solutions.

\section{Conclusions}

We have presented a model of baryogenesis which may lead to baryo-symmetric universe with cosmologically large domains of matter and antimatter, avoiding the domain wall problem. Breaking of charge symmetry is induced by a non-zero amplitude of a scalar field $\chi$, which slowly relaxed down to equilibrium, much slower than the process of baryogenesis goes on. In classification of different types of $C P$ violation which might be operative in cosmology this type is called dynamical one [24]. Later, after the baryon asymmetry was developed, $\chi$ evolved down to the equilibrium point $\chi=0$ and thus domain walls disappeared rather early in the universe. Inflation is an essential ingredient of the scenario. A coupling to the inflaton field was introduced on purpose to generate a non-zero value of $\chi$ and to keep it non-zero during baryogenesis.

This scenario inspired us to consider time evolution of thick domain walls in a de Sitter universe. We have shown that for large values of parameter $C>2$ the initial kink configuration in a de Sitter background tends to the stationary solution obtained by Basu and Vilenkin [12].

For $C<2$ the stationary solution does not exist and the width of the wall infinitely grows with time. For $C \lesssim 0.1$ the rise is close to the exponential one within the precision of our numerical calculations. This result confirms the assertion made in ref. [14] that the transition region between matter and antimatter domain might be cosmologically large. This result is essential for application of spontaneous breaking of symmetry between particles and antiparticles to realistic cosmology.

We acknowledge support of the Grant of President of Russian Federation for the leading scientific Schools of Russian Federation, NSh-9022-2016.2. SG is also supported under the grants RFBR No. 16-32-60115, 16-32-00241, 16-02-00342. In addition, SG is grateful to Dynasty Foundation for support.

\section{References}

[1] A.D. Dolgov, Phys. Rept. 222, 309 (1992)

[2] A.D. Dolgov, Surv. High Energ. Phys. 13, 83 (1998)

[3] V.A. Rubakov and M.E. Shaposhnikov, Phys. Usp. 39, 461 (1996) 
[4] A. Riotto and M. Trodden, Ann. Rev. Nucl. Part. Sci. 49, 35 (1999)

[5] M. Dine and A. Kusenko, Rev. Mod. Phys. 76, 1 (2003)

[6] A.G. Cohen, A. De Rujula and S.L. Glashow, Astrophys. J. 495, 539 (1998)

[7] Ya. B.Zeldovich, I.Yu. Kobzarev and L.B. Okun, Sov. Phys. JETP 40, 1 (1974)

[8] V.A. Kuzmin, I.I. Tkachev and M.E. Shaposhnikov, Pisma Zh. Eksp. Teor. Fiz. 33, 557 (1981)

[9] V.A. Kuzmin, M.E. Shaposhnikov and I.I. Tkachev, Phys. Lett. B 105, 159 (1981)

[10] V.A. Kuzmin, M.E. Shaposhnikov and I.I. Tkachev, Phys. Lett. B 105, 167 (1981)

[11] V.A. Kuzmin, M.E. Shaposhnikov and I.I. Tkachev, Nucl. Phys. B 196, 29 (1982) [Erratum ibid. B 202, 543 (1982)]

[12] R. Basu and A. Vilenkin, Phys. Rev. D 50, 7150 (1994)

[13] N.A. Voronov, A.L. Dyshko and N.B. Konyukhova, Phys. Atom. Nucl. 68, 1218 (2005)

[14] A.D. Dolgov, S.I. Godunov, A.S. Rudenko and I.I. Tkachev, JCAP 10, 027 (2015)

[15] A.D. Dolgov, S.I. Godunov, A.S. Rudenko, JCAP 10, 026 (2016).

[16] D.S. Gorbunov, V.A. Rubakov, Introduction to the theory of the early universe: Cosmological perturbations and inflationary theory, World Scientific (2011).

[17] V.A. Kuzmin and I.I. Tkachev, Phys. Rev. D 59, 123006 (1999)

[18] Yu.V. Shtanov, J.H. Traschen and R.H. Brandenberger, Phys. Rev. D 51, 5438 (1995)

[19] L. Kofman, A.D. Linde and A.A. Starobinsky, Phys. Rev. Lett. 73, 3195 (1994)

[20] L. Kofman, A.D. Linde and A.A. Starobinsky, Phys. Rev. D 56, 3258 (1997)

[21] M. Kobayashi and T. Maskawa, Prog. Theor. Phys. 49, 652 (1973)

[22] D.V. Nanopoulos and S. Weinberg, Phys. Rev. D 20, 2484 (1979)

[23] A.D. Sakharov, JETP Lett. 5, 24 (1967); Sov. Phys. Usp. 34 (1991) 392

[24] A.D. Dolgov, CP violation in cosmology, in the proceedings of 163rd Course of International School of Physics 'Enrico Fermi': CP Violation: From Quarks to Leptons, Varenna, Italy, July 19-29, 2005 [hep-ph/0511213]. 